\title{
Tremor, the cogwheel phenomenon and clonus in Parkinson's disease
}

\author{
L J FINDLEY, M A GRESTY, AND G M HALMAGY I*
}

From the Medical Research Council Hearing and Balance Unit, Institute of Neurology, National Hospital, Queen Square, London and the Department of Clinical Neurophysiology,* Royal Prince Alfred Hospital, Camperdown NSW, Australia

SUMMARY Resting and postural tremor, intention and action tremor, clonus and the cogwheel phenomenon in Parkinson's disease have been characterised in terms of frequency content using spectral analysis. Typical resting tremor ranged in peak frequency from 4 to $5 \cdot 3 \mathrm{~Hz}$ with tremor in each individual varying only by 0.2 to $0.3 \mathrm{~Hz}$. The peak frequency of postural tremor ranged between 6 and $6.2 \mathrm{~Hz}$. Intention tremor appeared to be an exaggeration of postural tremor. Clonus evoked by active or passive stretch at the wrist had a frequency of $6 \mathrm{~Hz}$ and appeared to be a continuation of postural tremor. The cogwheel phenomenon was found at frequencies between 6 and $6.5 \mathrm{~Hz}$ and between 7.5 to $9 \mathrm{~Hz}$. Action tremor was indistinguishable from the cogwheel phenomenon. Some patients had either a symptomatic resting tremor with a concurrent $6 \mathrm{~Hz}$ component of smaller amplitude or a symptomatic postural tremor with a 4-5 Hz component of smaller amplitude. These combinations would produce two peaks in the power spectrum. When this occurred EMG studies showed that individual muscles had two types of rhythmical activation suggesting that the tremors have separate mechanisms. Likewise some patients had a symptomatic $6 \mathrm{~Hz}$ tremor on posture with a second peak at $8-10 \mathrm{~Hz}$ in the physiological band. Therefore, the $6 \mathrm{~Hz}$ postural tremor is not an exaggeration of physiological tremor. On the basis of wave form and frequency similarities postural tremor, the low frequency type of active or passive cogwheeling, intention tremor and clonus possibly involve a common spinal mechanism. Higher frequency cogwheel phenomenon and action tremor may be an exaggeration of physiological tremor. More than $80 \%$ of patients with Parkinson's disease manifest tremors at both $4-5 \mathrm{~Hz}$ and $6 \mathrm{~Hz}$. This combination would appear to be the strongest objective criterion for the diagnosis of basal ganglia disease.

There are three forms of tremulous movement in Parkinson's disease, the resting tremor, action and postural tremor, and the cogwheel phenomenon.

Parkinson $^{1}$ re-emphasised that resting tremor was a dominant feature in this disease, since when there have been numerous descriptions of its characteristics. ${ }^{2-4}$ Frequently commencing in fingers and thumb on one side and later involving lips, tongue and the ipsilateral ankle. It is usually distal, and is seen with the limb at rest ceasing before or with the onset of voluntary movement. As the disease progresses, however, it may be present throughout action and can be maintained

Address for reprint requests: Dr MA Gresty, MRC Hearing and Balance Unit, The National Hospital, Queen Square, London, WC1 - 3BG. Accepted 12 February 1981 in posture. Resting tremor is reported between 4 and $7 \mathrm{~Hz}$, and results from the reciprocal contraction of antagonistic muscle groups. ${ }^{5}$ The origin of this rhythmical involuntary activity is still not clear, ${ }^{56}$ but degeneration of the nigro-striatal pathway and depletion of dopamine in the striatum are the most constant central features required for the symptoms of Parkinson's disease to occur. ${ }^{7}$

Lewy ${ }^{8}$ discussed the appearance of a static (that is postural) and locomotorial action tremor in paralysis agitans. De Jong ${ }^{9}$ distinguished action from intention tremor, and his recordings showed a tremor throughout movement which was continuous with the tremor that occurred when the limb was maintained in posture. Lance et $a^{10}$ found marked action tremor in 17 of 33 patients 
with Parkinson's disease who had symptomatic resting tremor, and in six out of seven patients without resting tremor. The frequency range of action tremor was 7 to $12 \mathrm{~Hz}$ and it was shown that this tremor arose through co-contraction of active muscles, and was thus distinct from the resting tremor. Lance ${ }^{10}$ and his colleagues thought that this tremor was an exaggerated physiological tremor inderendent of the stretch reflex arc or gamma efferent activity. In addition to the common tremors, there is recent evidence in Parkinson's disease that there can be an associated intention tremor, that is, an exaggeration of tremor brought out at the termination of movement, such as during the finger to nose test. This was interpreted as being due to exaggeration of postural tremor. ${ }^{19}$

Rigidity as a feature of the disease is not mentioned in Parkinson's original monograph. ${ }^{1}$ The term "cogwheel phenomenon" has been attributed to Camillo Negro, who first described it in $1901,{ }^{11}$ though the first description in English literature is that of Moyer in 1911. ${ }^{12}$ Lance $^{10}$ elicited the cogwheel phenomenon during passive movements of the hand at the wrist joint at frequencies ranging more often between 6 and $12 \mathrm{~Hz}$ than from 3 to $6 \mathrm{~Hz}$. He concluded that either the resting or the action tremor was responsible for what was interpreted to be a segmentation of a hyperactive stretch response to give the typical "cogwheel effect". This concept of the origin of the "cogwheel phenomenon" was supported by Selby, ${ }^{2}$ though Denny-Brown ${ }^{3}$ suggested that both rigidity and tremor could be attributed to the same underlying neuronal mechanism. The latter arises because this mechanism is unstable. The origin of rigidity also is not certain, particularly as to whether the final common pathway is via the gamma efferent system, ${ }^{13} 14$ the alpha motorneurones, ${ }^{15}$ or both ${ }^{16}{ }^{17}$; a summary of current views is given by Evarts. ${ }^{18}$

Finally, there are occasional reports that sustained passive stretch in extension or flexion of the wrists or fingers in some patients produces a powerful regular tremor which we find is clinically indistinguishable from clonus and feels as if it is a continuation of the cogwheel phenomenon produced during the dynamic phase of muscle stretch. This has been recognised in all stages in the natural history of the disease. ${ }^{3}$

The present survey of the rhythmical involuntary movements of Parkinson's disease has utilised techniques of wave form analysis in an attempt to make a more precise and simple classification of the diverse phenomena involved. The primary concern has been a phenomenological analysis of distal tremor as transduced by movement detectors attached externally to the limb. Surface electromyograms were performed in order to relate the wave forms of tremor to muscle activity. Only movements in flexion and extension at the wrist have been considered; and because the amplitude of the tremors in Parkinson's disease fluctuate so markedly, attention has been given only to whether tremor was symptomatic or not.

\section{Subjects and methods}

Patients with idiopathic Parkinson's disease (mean age $64 \pm 8 \cdot 1$ (1SD) years, and mean duration of symptoms $5.9 \pm 4.2$ (1SD) years) were studied in the MRC Hearing and Balance Unit at the National Hospital for Nervous Diseases, Queen Square. The first 40 of these patients were included in the survey of resting and postural tremor, the results of which are presented in the frequency histograms in fig 1 . More than 20 additional patients were investigated during the preparation of the manuscript. Thirty patients were taking levodopa in a combined tablet with a peripheral decarboxylase inhibitor carbidopa (Sinemet); the average daily dose was $370 \mathrm{mg}$ (range 110 to $825 \mathrm{mg}$ ). Six of the levodopa group and three of the other patients were taking anticholinergic drugs. Drug treated patients were all studied in the morning, having discontinued their medication on the previous evening. An initial pilot study of patients with Parkinson's disease confirmed that levodopa had no effect on the frequency of tremor and, in keeping with the findings of others, did not always attenuate the amplitude. ${ }^{20}$ Many patients were studied on several occasions.

The subject was seated in a chair with broad flat arms, each covered with a sheet of foam rubber to dampen artefacts produced by the arm resting on a "pulse." Tremor at rest was measured with the subject's forearms lying pronated along the arms of the chair with the hands dangling freely over the edges. Tremor in posture was measured with the hands elevated to a horizontal position, gently stretching out beyond the arms of the chair with the subject's forearms still supported in their length by the armchair. Cogwheel phenomena were evoked passively and actively. In the active situation the subject was seated as above with hands extending beyond the arms of the chair; to evoke cogwheeling the wrists were flexed and extended throughout their range of movement as rhythmically as possible, each cycle taking typically two to three seconds. The examiner palpated each wrist gently during this manoeuvre. Passive cogwheeling was evoked by the examiner flexing and extending the subject's hand whilst gently palpating the subject's wrist. Clonus was evoked either by the examiner manipulating the wrist in flexion/ extension or supination/pronation in order to induce stretch, or by the subject pressing in flexion with his fingers on to a strain gauge mounted beyond the edge 

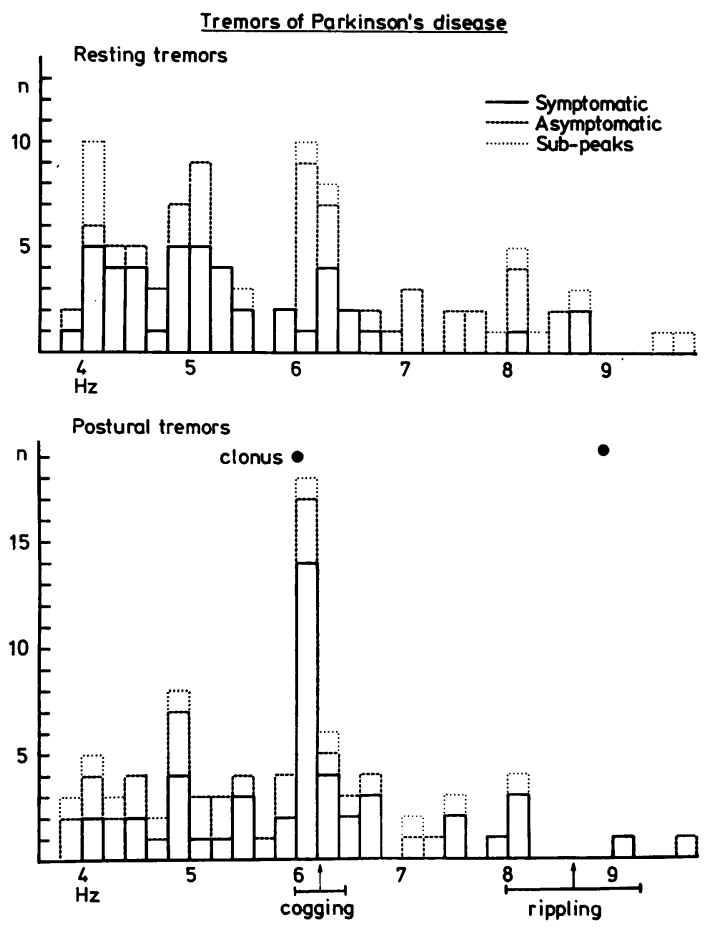

of the arm of the chair and thus inducing tendon stretch.

Tremulous and vibratory movements were transduced using linear accelerometers and the Schottky barrier photodetector. In transducing tremor the accelerometer was mounted on the dorsum of the hand with its sensitive axis in the plane of flexion/extension or pronation/supination. A target light for the Schottky barrier photodetector was attached to the body of the accelerometer. Accelerometers used were linear piezo-resistive devices with a flat frequency response from DC to at least $200 \mathrm{~Hz}$ and sensitive to at least $1 / 1000$ th of $g$. All recordings were calibrated absolutely with respect to gravity. The Schottky photodetector was used to transduce limb displacement. The detector was mounted in the focal plane of a camera which was positioned to view the limb from a suitable angle, in this case viewing the light placed on the dorsum of the hand from the side. The detector gives a read out in $\mathrm{x}$ and $\mathrm{y}$ co-ordinates of the target light. ${ }^{19}$ Recordings were presented on a storage oscilloscope and a $\mathrm{y} / \mathrm{t}$ electrostatic ink jet recorder. Surface electromyograms were taken using silver/silver chloride cup electrodes of $4 \mathrm{~mm}$ diameter, placed $2 \mathrm{~cm}$ apart. Accelerometric signals were processed "on line" using a digital computer to calculate power spectra. They were shaped using a high pass filter with a time constant of 1 second and a low pass filter with a fall off of $6 \mathrm{~dB}$ per octave having an
Fig 1 Frequency histograms of resting and postural tremors in the individual limbs of 40 patients with idiopathic Parkinson's disease. Upper histogram: typical gross resting tremor characteristic of the disease, occupying frequency band 3.8 to $5.6 \mathrm{~Hz}$. Above $5.8 \mathrm{~Hz}$ are faster tremors of smaller amplitude, more akin in appearance to the typical postural tremor of Parkinsonism although present with limb in a resting position. Lower histogram: postural tremor at frequencies below $5.6 \mathrm{~Hz}$ had the visible characteristics of the typical resting tremor although the limb was in posture, whereas those above $5.8 \mathrm{~Hz}$ had the appearance of the typical fine fast tremor of Parkinsonism. The envelope of asymptomatic tremor frequencies closely follows the distribution of symptomatic tremors suggesting analysis of the asymptomatic tremor may have diagnostic value. Sub peaks on the power spectra which were not harmonically related to the dominant peak frequency are plotted in the histograms and these are also predominantly distributed around the modal values for the frequencies of symptomatic tremor. Inserts in the lower histogram show the coincidence of frequencies cogwheeling, clonus and typical symptomatic postural tremor. The bars indicate the absolute range of frequency values obtained for cogwheeling and rippling.

elbow at $16 \mathrm{~Hz}$, then digitised with a $10 \mathrm{~ms}$ sampling rate, windowed with a hamming function and subjected to a 1024 point Fourier decomposition from which auto spectra and power spectra from DC to $25 \mathrm{~Hz}$ and a dynamic range of $48 \mathrm{~dB}$ were calculated.

In Parkinson's disease the typical resting tremor in a limb is $4 \mathrm{~Hz}$ and when the limb lifts into posture a tremor at $6 \mathrm{~Hz}$ becomes manifest; does this mean that the tremor generating mechanism has speeded up? We will argue that the two frequencies of tremor are generated by different mechanisms, but one can only argue this on the basis of spectral analysis if strict criteria are met. Firstly one must show that tremor at the two frequencies can occur together producing two harmonically unrelated peaks in the power spectrum. Secondly, when this occurs it must be due to the fact that the tremors run concurrently and do not alternate. As tremor of the hand involves several muscles, each of which may be supporting a different tremor frequency and the accelerometer transduces the net sum of these as if they were a singlemechanism, the presence of harmonically unrelated peaks in the power spectrum of a tremor is not itself sufficient to make the conclusion that separate physiological mechanisms are involved. Accordingly in cases of compound tremor we examined the activity in single muscles by electromyography to establish that the patterns of electrical activity also showed that more than one type of periodic activation was present. 


\section{Results}

RESTING AND POSTURAL TREMORS

Amplitude of tremor

Tremor amplitude was simply characterised as causing symptoms or not. In terms of amplitude, in the case of a $6 \mathrm{~Hz}$ tremor frequency, a peak acceleration of 0.01 to $0.1 \mathrm{~g}\left(\mathrm{~g}=981 \mathrm{~cm} \mathrm{~s}^{-2}\right)$ were moderately symptomatic. Acceleration amplitudes above this range caused severe symptoms and accelerations below this range were not symptomatic.

Frequency analysis of tremor

Each tremor was characterised in terms of its dominant frequency and also in terms of harmonically unrelated peaks which were within $8 \mathrm{dBs}$ of the dominant peak in amplitude. Asymptomatic tremors were characterised only in terms of the dominant frequency present. The frequency parameters obtained in this way were plotted in histogram form presented in fig 1 . The columns of the histogram were selected to be $0.2 \mathrm{~Hz}$ wide. This resolution was chosen because, both within recording sessions and from session to session, the frequency of a symptomatic tremor was never seen to vary more than about $0 \cdot 3 \mathrm{~Hz}$. In fact, we have found the constant frequency of pathological tremors in individual subjects to be a useful criterion to distinguish between normal and pathological tremor.

\section{Resting tremors}

As illustrated in fig 1, the majority of symptomatic resting tremors in Parkinson's disease have peak frequencies which lie between 4 and $5.3 \mathrm{~Hz}$. A smaller grouping lies between 5.8 and $6.8 \mathrm{~Hz}$. The lower frequency group is further divided between a concentration around 4 to $4.6 \mathrm{~Hz}$ and a concentration from 4.8 to $5.4 \mathrm{~Hz}$. There is a distinct discontinuity in the tremor spectrum between 5.6 and $5.8 \mathrm{~Hz}$. The addition of asymptomatic and subsidiary tremor frequencies to the histogram only accentuates the frequency distribution already determined by the symptomatic tremors. The tremors with frequencies from 4 to just above $5 \mathrm{~Hz}$ are those which were clinically identifiable as the classic resting tremors of Parkinson's disease (fig 2). The discontinuity in the histogram corres-
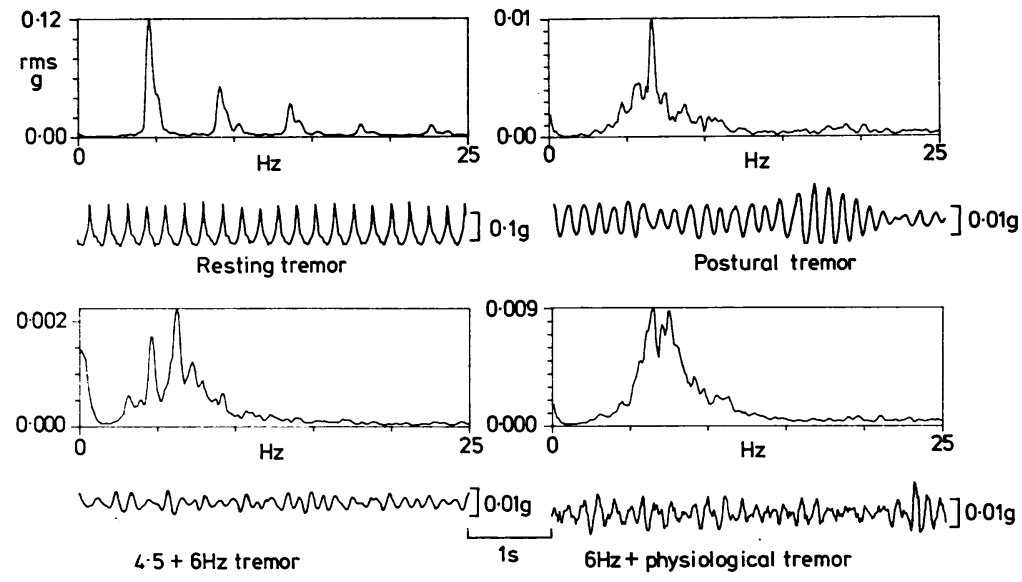

Fig 2 Auto-spectra of resting and postural tremors of the hand in Parkinson's disease. The top left spectrum shows analysis of a fairly pure resting tremor. The tremor has a dominant frequency between 4 and $5 \mathrm{~Hz}$, with 2nd, 3rd, 4 th and 5th harmonics. The top right spectrum is of pure postural tremor with a dominant frequency of 6 to $6.5 \mathrm{~Hz}$. The bottom left spectrum is of a resting tremor with dominant frequencies at 4 and $5 \mathrm{~Hz}$ and 6 to $7 \mathrm{~Hz}$. The concurrence of these two frequency components which are not harmonically related indicates that there are two coincident tremors. The bottom right spectrum is of a very fine postural tremor, with two peaks, one at $6 \mathrm{~Hz}$, the other at a higher frequency about $7.5 \mathrm{~Hz}$ which, by similar argument as before, indicates two separate tremor mechanisms. In this case the $6 \mathrm{~Hz}$ is probably the pathological postural tremor of the disease, whereas the higher frequency component is the patient's normal physiological tremor. 
ponds to the division between what are, on appearance, typically resting and typically postural tremors. That is, in some patients the typical postural tremor may also be evident in the resting position.

\section{Postural tremors}

The frequencies of postural tremors are predominantly grouped around 6 to $6 \cdot 2 \mathrm{~Hz}$ and these represent the fine typical postural tremor of the disease (fig 2). The incidences of tremor around 4 to $5 \mathrm{~Hz}$ reflect the observation that occasionally the resting tremor of the disease may continue when the limb is raised in posture.

Amongst our patients we observed a typical symptomatic resting tremor in 31 limbs at rest and in 10 limbs on posture. Conversely the typical higher frequency symptomatic postural tremor was observed in 25 limbs on posture and in 10 at rest. We may conclude from the comparable incidences of the two types of tremor in this disease that they are almost equally characteristic of the condition.

\section{Differentiation of resting, postural and}

physiological tremor in power spectra and EMG Analysis of a tremor commonly produced a power spectrum with more than one peak, the peaks being harmonically unrelated. A resting tremor at $4-5 \mathrm{~Hz}$ sometimes also contained a frequency component at $6 \mathrm{~Hz}$; alternatively a postural tremor might consist of components at both $6 \mathrm{~Hz}$ and $8-9 \mathrm{~Hz}$ (fig 2). This finding suggests that more than one frequency of tremor was being supported in the limb. EMG studies running concurrently with the tremor analysis revealed that the multiple tremors could also arise from one muscle at the same time and evidence for this is presented in fig 3 . The recordings were taken over the belly of the extensor digitorum in the right forearm of one patient with the hand in rest and posture. In each condition there were at least two types of EMG activity present. At rest there was a large amplitude double spike packet at about $4 \mathrm{~Hz}$ with a smaller, more ragged higher frequency burst of activity which averaged $6 \mathrm{~Hz}$. In posture there were less regular large amplitude single spiked packets with a single large spike at $6.7 \mathrm{~Hz}$ together with a much smaller burst of activity at about 8 to $9 \mathrm{~Hz}$, which was interpreted as being "normal" physiological activity.

Although interpretation of the EMG activity was difficult on occasions, mixed types of activity were found in individual wrist and hand muscles of patients who had tremors which decomposed into
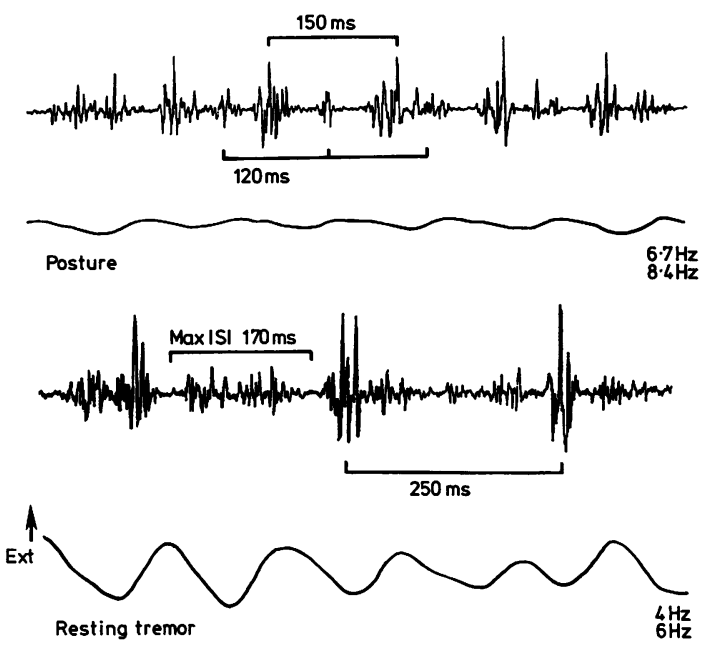

Fig 3 Surface EMG recordings over the belly of extensor digitorium in right forearm of a patient with idiopathic Parkinson's disease. Tremor wave forms were recorded with an accelerometer mounted on the dorsum of the hand. In posture there are large bursts of activity characterised by a dominant single spike at an average frequency of $6.7 \mathrm{~Hz}$ together with smaller bursts of activity at an average frequency of 8 to $9 \mathrm{~Hz}$. Spectral analysis of the tremor wave form also revealed similar distinct frequencies. At rest there are large double spiked packets of activity at $4 \mathrm{~Hz}$ superimposed upon background bursts of activity at about $6 \mathrm{~Hz}$. When bursts of different tremor activities are superimposed in this way it can be difficult to estimate individual frequencies in which case a useful parameter is the maximum inter-silent interval (Max ISI) which corresponds to the highest frequency tremor present. These results demonstrate that both at rest and in posture separate myogenic tremor mechanisms may co-exist.

more than one dominant frequency. This finding supports the hypothesis that the resting, symptomatic postural and physiological tremors are separate entities, generated by different mechanisms. In the analysis of postural tremors, five limbs with symptomatic tremor and two with asymptomatic tremor had significant peaks at both 6 and 8 to $9 \mathrm{~Hz}$. Seven limbs with symptomatic tremor and five with asymptomatic tremor had significant peaks at 4 to 5 and $6 \mathrm{~Hz}$. Two limbs with symptomatic tremor had peaks on the power spectrum at all three frequencies. Amongst resting tremors; one limb had symptomatic and five had asymptomatic tremor with peaks at 6 and 8-9 $\mathrm{Hz}$, four had symptomatic and six asymptomatic tremor with peaks at $4-5$ and $6 \mathrm{~Hz}$ and four 
limbs, all with symptomatic tremor, had peaks at all three frequencies.

\section{Asymptomatic tremors}

The distribution of peak frequency of asymptomatic tremor was similar to that of the symptomatic tremors in many patients (fig 1), implying that tremor analysis could help in the detection of early Parkinsonism in patients where there was no symptomatic tremor.

\section{Incidence of symptomatic postural and resting}

tremors

Regardless of the dominant frequency of tremor, amongst the 40 patients whose results are presented in fig 1, 44 limbs had "typical" symptomatic resting tremor, and 44 limbs had "typical" symptomatic postural tremor. Thirteen limbs had symptomatic tremors which occurred with a similar appearance in both rest and posture; the tremors were both of the $6 \mathrm{~Hz}$ postural type in four, and a further four were of the $4-5 \mathrm{~Hz}$ resting type; the remaining five showed both types of tremor symptomatically. The concurrence of the classic symptomatic resting tremor of Parkinsonism with symptomatic postural tremor in the same limb was rare. Far more common was that one particular form of tremor, the 4-5 $\mathrm{Hz}$ type or the $6 \mathrm{~Hz}$ type, predominated in "appearance," and on spectral analysis was found to have a second frequency present corresponding to the other type of tremor.

COGWHEELING PHENOMENON

We adopted the following strict criteria which a record of cogwheeling had to fulfil in order to be subject to frequency measurements. Firstly the examiner had to "feel" the jerks of cogwheel rigidity occurring in a regular run, and at the same time identify the corresponding parts of the movement recording on the oscilloscope or ink jet recorder. Secondly, this part of the record then was examined for similar wave forms which repeat without interruption, thus establishing

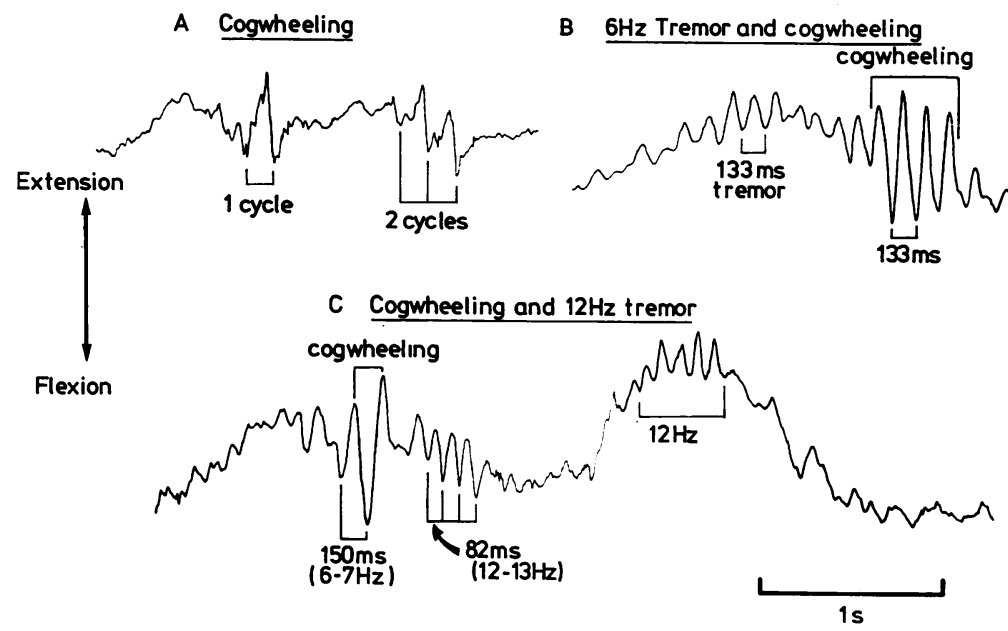

Fig 4 Examples of the most typical wave forms of cogwheel phenomenon. The recordings were taken with an accelerometer mounted on the dorsum of the hand, which was passively moved in flexion and extension. Upper right " $A$ " illustrates a "sawtooth" shaped wave form; the rapid down stroke indicates that the strongest contraction is in the flexors. Later in the trace, when two cycles of cogwheeling appear with similar wave forms, the phenomenon has the appearance of a transient tremor and can be measured in terms of frequency $(6 \mathrm{~Hz})$. The upper right hand traces " $B$ " illustrate $a$ sinusoidal cogwheel wave form which is well developed for several cycles. In this patient, throughout all posture and movement, a $6 \mathrm{~Hz}$ tremor persisted. The cogwheel phenomenon appears to be a continuation and exaggeration of the tremor. The traces illustrated in " $C$ "' show a strong beat of cogwheeling at 6-7 $\mathrm{Hz}$ occurring with a much faster transient tremor at $12-13 \mathrm{~Hz}$, which may represent an apparent frequency doubling of the basic tremor through enhancement of the second harmonic. 
periodicity. Frequency measurements then were made from the repetition rate of the wave form. Frequencies of individual samples of cogwheeling were based on a mean taken over as many cycles as possible. Cogwheeling which occurred at a harmonic of a lower frequency present in the record was classified with the fundamental (fig 4C). Applying these criteria, we have encountered wave forms which correspond to the clinical appreciation of cogwheeling at two distinct frequencies, $6 \mathrm{~Hz}$ and 8 to $9 \mathrm{~Hz}$, without overlap.

\section{Cogwheel phenomenon at $6 \mathrm{~Hz}$}

Fifteen of our patients cogwheeling which had periodic wave forms with frequencies between 6 and $7 \mathrm{~Hz}$. The mean frequency was 6.22 (SD) $\pm 0 \cdot 137) \mathrm{Hz}$, indicating that the frequency distribution was biased towards $6 \mathrm{~Hz}$, reminiscent of postural symptomatic tremor. At times the wave form of cogwheeling appeared to be sinusoidal, much like an exaggeration of ongoing postural tremor (at around $6 \mathrm{~Hz}$ ) (fig 4B, C). At other times the wave form was more sawtooth in shape (fig 4A).

Cogwheel phenomenon at 8 to $9 \mathrm{~Hz}$ - "rippling" Eighteen of our patients exhibited cogwheeling which ranged in frequency between 7.5 and $9 \mathrm{~Hz}$, with a mean of $8.6(\mathrm{SD} \pm 0.7) \mathrm{Hz}$ (fig 5). This type of resistance to passive movement felt smoother and more regular than lower frequency cogwheeling, and invariably had a sinusoidal wave form; "rippling" describes fairly well how it felt. Cogwheeling at $6 \mathrm{~Hz}$ and rippling at $8-9 \mathrm{~Hz}$ were not
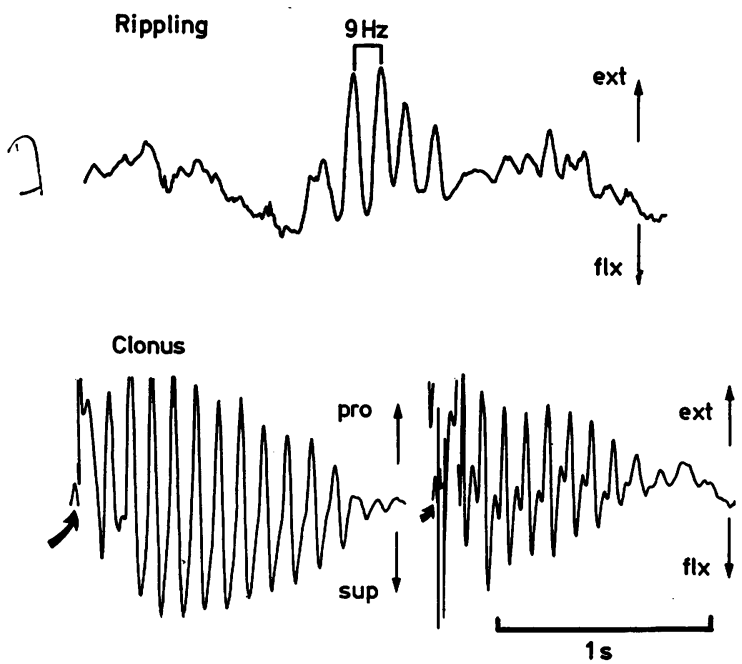

mutually exclusive phenomena; both occurred in four limbs in our patients in the same recording session. Cogwheeling or rippling evoked passively differed little from that brought out actively by the patient himself.

COGWHEEL PHENOMENON AND CLONUS

In six of our original survey of 40 patients, and in 10 others examined subsequently, we found that during manipulations to evoke cogwheel phenomena, sustained stretching of the wrist evoked a vibration which was clinically indistinguishable from clonus. Moreover, on the occasions when we were able to record symptomatic postural tremor, cogwheeling with regular wave form, and clonus in the same limb during a single recording session, all three phenomena were at the same frequency with similar wave forms. These similarities are illustrated in the recordings of fig 6 which were taken from a 56-year-old patient with a year long history of idiopathic Parkinson's disease. The resting tremor of his right hand had a frequency of $4.5 \mathrm{~Hz}$. Postural tremor of the hand was at $6.3 \mathrm{~Hz}$. There was also the cogwheel phenomena and an action tremor present at frequencies between 6 and $6.3 \mathrm{~Hz}$, while wrist extension produced clonus at $6 \cdot 1 \mathrm{~Hz}$. The cogwheel phenomena and clonus tended to be well developed in patients with a symptomatic postural tremor, and in all but one such patient cogwheeling was at the same frequency as the tremor.

Patients with Parkinsonism and clonus of the upper limb did not have "pyramidal" signs, but we did examine clonus in other diseases. The patients included a 17 -year-old male with cervical cord transection due to trauma, a 50-year-old male with cervical myelopathy, two adult males

Fig 5 "Rippling." An example of high frequency cogwheeling recorded from a patient with idiopathic Parkinson's disease. The transient tremor is typically sinusoidal. The lower traces are of clonus in extension and pronation at the wrist recorded from a patient with an extensive brain stem angioma. The clonus in extension-flexion is similar in wave form and frequency to the rippling and the clonus in supination-pronation is similar to that of "sawtooth" shaped cogwheeling as illustrated in fig 3. The arrows indicate the point at which the hand was manipulated by the examiner. These records were obtained using an accelerometer mounted on the dorsum of the hand with its sensitive axis in the plane of movement. The same relative gains are maintained throughout. 

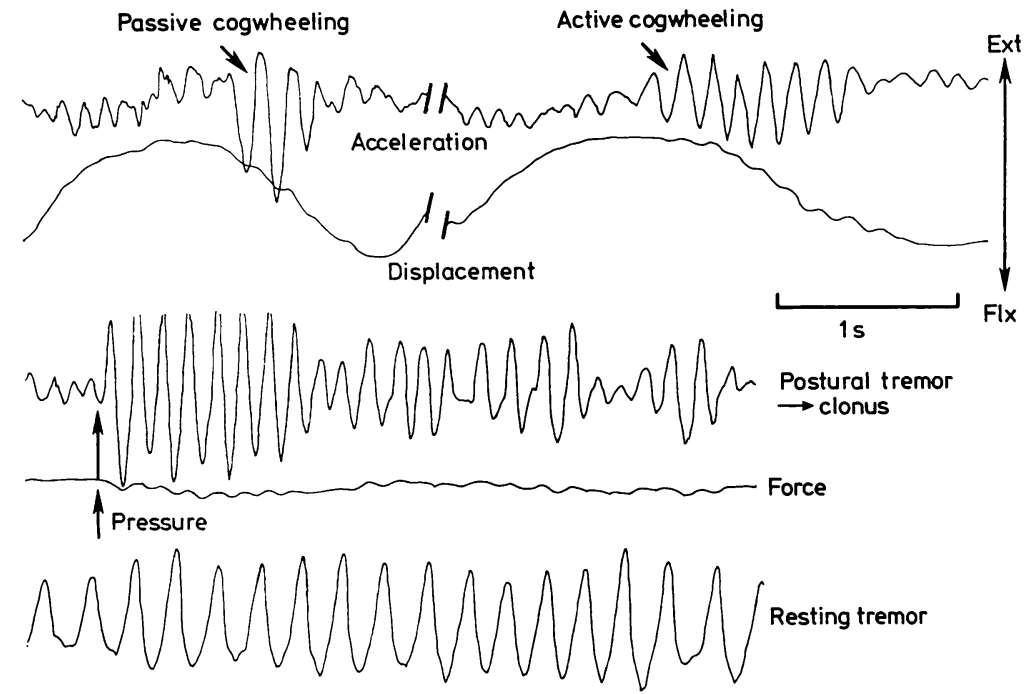

Fig 6 Recordings of cogwheeling, active cogwheeling (action tremor), resting tremor, postural tremor and clonus taken from a patient with idiopathic Parkinson's disease. The records were all taken in the same recording session within a few minutes of each other. The traces of the upper left show acceleration and displacement records of passive cogwheeling evoked with a frequency of $6 \mathrm{~Hz}$. A few seconds later the patient was asked to move his own hand in flexion and extension, as in the records of the upper right, evoking a more sustained tremor at $6 \mathrm{~Hz}$ with similar wave form. The middle traces are of accelerometric recordings of the hand tremor in posture which goes into clonus as the patient presses down (hand being extended) on a force platform. The arrows indicate the point at which he begins to press on the platform. The frequency of clonus is $6 \mathrm{~Hz}$. In the lowest trace the hand is at rest and develops a typical $4.3 \mathrm{~Hz}$ resting tremor. A constant relative gain on the accelerometer traces was maintained throughout.

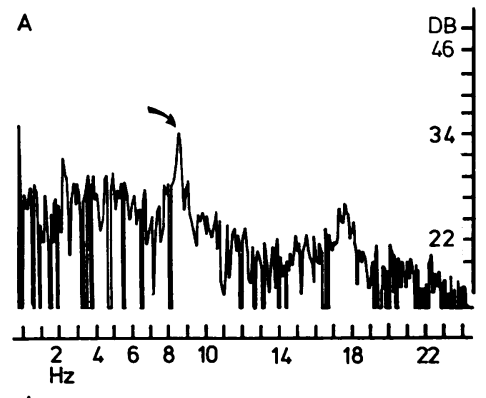

A

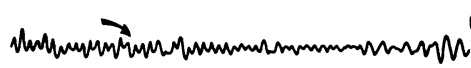

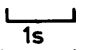

Exaggerated physiological tremor in corfical infarct
B

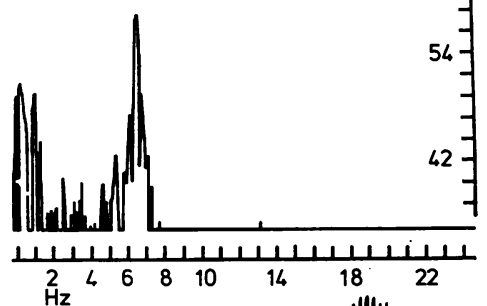

B

$0 \cdot 19$

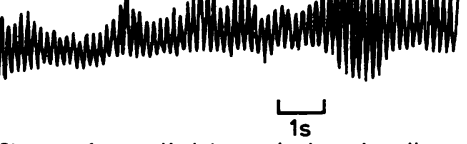

Fig 7 Raw data records and corresponding power spectra of a fine $9 \mathrm{~Hz}$ sinusoidal tremor, presumed to be an exaggeration of physiological tremor in cortical infarct and of clonus at $6 \mathrm{~Hz}$ in a patient with a cervical cord lesion. The raw data records are of comparable amplitudes. The arrows in " $A$ " indicate the sections of the raw data record which have a measurable frequency corresponding to the finely tuned peak of the power spectrum. 
with a cerebral cortical infarcts and a 27 -year-old male with a brain stem angioma. The first and last patients had clonus on passive supination and pronation, and on flexion and extension of the wrist; the remaining patients had clonus on both passive stretch and during maintained arm posture. As with cogwheel phenomenon, we found two frequencies at which clonus occurred. The patient with a cervical myelopathy had clonus at $6 \mathrm{~Hz}$; the patient with a cortical infarct also had clonus somewhat less well sustained at $9 \mathrm{~Hz}$ (fig 7). The patient with an angioma had transient clonus in both supination and pronation and flexion at $9 \cdot 2 \mathrm{~Hz}$ (fig 5), and the patient with a cord section had transient clonus in pronation at $9 \mathrm{~Hz}$. Thus there was a parallel between the two forms of cogwheeling at $6 \mathrm{~Hz}$ and 8 to $9 \mathrm{~Hz}$, and the two frequencies at which we have demonstrated clonus. The greatest differences between the cogwheel phenomenon and clonus was that clonus almost invariably had a more regular wave form and was often a more powerful tremor lasting longer (figs 5 and 6).

Postural tremor of the hand in normal subjects The possibility that symptomatic postural tremor of the hand in people with Parkinson's disease represented an exaggeration of normal physiological tremor led us to survey the characteristics of postural tremor in normal adults. Ninety-five normal drug-free subjects were examined to exclude any significant neurological lesions and each individual was subjected to six spectral analyses of tremor of each hand in posture. Normal postural tremor of the hand in subjects of all ages was often broadly tuned with several peaks of similar amplitude making characterisation in terms of a single frequency difficult. Moreover the overall shape of the spectra derived changed frequently from one tremor sample to the next. In order to characterise the variability of tremor frequency the following measurements were adopted. For the six recordings from each hand the lowest tremor frequencies were identified. These were then combined and averaged over all hands and subjects in successive 10 year age groups. The same was done separately for the highest peak frequencies found. The means and standard deviations thus calculated were plotted as a function of age group (fig 8). Variability and range of dominant tremor frequency did not alter with age, although there was an overall decline in frequency in the oldest age group. There was no evidence of a convergence on $6 \mathrm{~Hz}$ tremor with age. These findings are in contrast to those

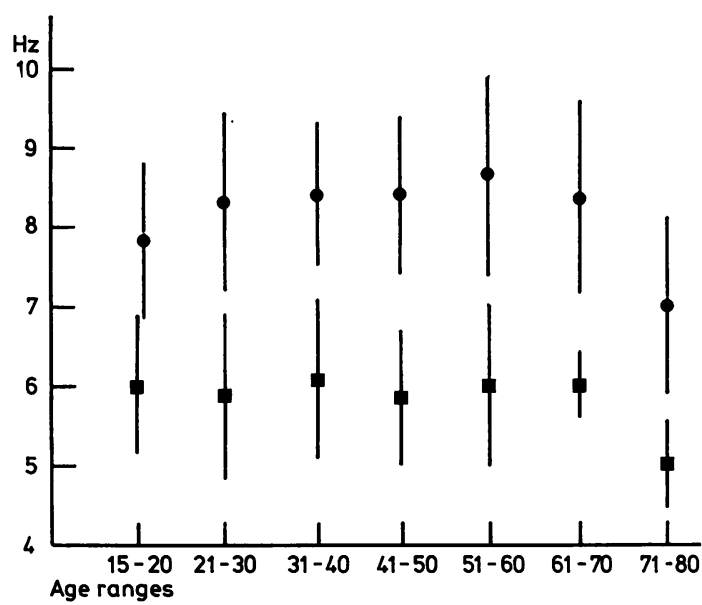

Fig 8 Means and standard deviations of the highest and lowest peak frequencies of tremor observed during six recordings of each limb on hands of 95 normal subjects plotted as a function of age groupings. The data were averaged over both hands and all subjects in each age group. There is no tendency for the variability of tremor frequency to change with age and no tendency for convergence on a $6 \mathrm{~Hz}$ tremor similar to the postural tremor of Parkinson's disease. There is a trend towards decreasing tremor frequencies with the most advanced age group.

in patients with Parkinson's disease in whom the postural tremor was relatively invariant in frequency and most commonly at $6 \mathrm{~Hz}$. Under certain circumstances normal subjects may show tremor characteristics similar to those of basal ganglia disease (fig 9). In fig 9A, an example of a broadly tuned postural hand tremor in a normal subject is presented. Although one could say that the dominant tremor frequency lay between 8 and $10 \mathrm{~Hz}$, there are obviously many more components of significance. The spectrum of figure 8B was derived from the same subject after performing vigorous isometric exercises with his hand and then holding it in posture whilst forcibly contracting his forearm muscles. These manoeuvres produced a more finely tuned tremor with a single dominant frequency at $6 \mathrm{~Hz}$; there is also a distinguishable sub-peak at $4 \mathrm{~Hz}$. The combination of fatigue and rigidity in this normal subject induced a pattern of tremor similar in frequency to the pathological tremors of basal ganglia disease. 

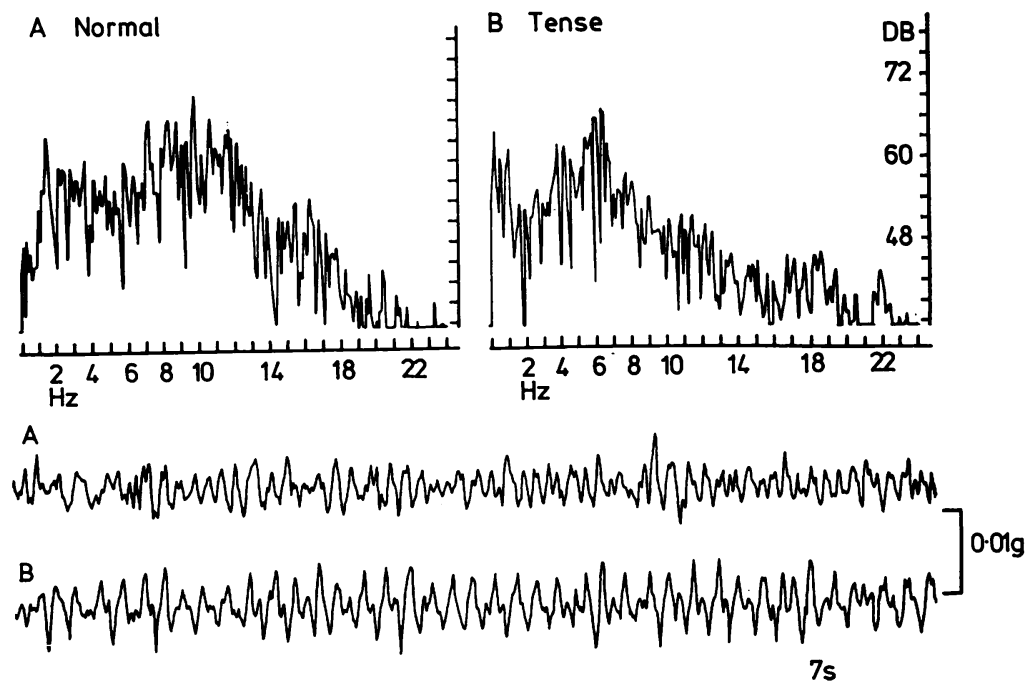

Fig 9 Raw data records and corresponding power spectra of tremor of the hand in posture recorded from a normal male subject aged $34 \mathrm{yr}$. " $A$ "- - The power spectrum of the tremor of normal posture is an example of a broadly tuned tremor. Although the peak value is clearly $10 \mathrm{~Hz}$ there are significant components between 7 and $12 \mathrm{~Hz}$ making it difficult to characterise the tremor in terms of a single frequency. (The high power content at $2 \mathrm{~Hz}$ probably represents postural adjustment.) " $B$ "- The subject has maintained isometric co-contraction of the hand muscles for several minutes. As shown in the raw records, the tremor has become more regular in appearance and the power spectrum shows a finely tuned peak at $6 \mathrm{~Hz}$. There is also a significant subsidiary peak at 4-5 Hz. Postural adjustments at low frequencies have increased in amplitude and there is probably an increased cardioballistic component. The raw data records are to scale.

\section{Discussion}

Different types of tremor in Parkinson's disease The apparently diverse tremors of Parkinson's disease can be grouped together into three frequency bands, which for convenience will be referred to as $4-5 \mathrm{~Hz}$ tremor, $6 \mathrm{~Hz}$ tremor, and 8-9 $\mathrm{Hz}$ tremor. Tremors in these three frequency bands are separate entities as shown by spectral analysis and EMG studies in individual muscles.

The classical resting tremor of Parkinson's disease had a fundamental frequency between 4 and $5.3 \mathrm{~Hz}$. The actual frequency in any individual patient varied only by 0.2 to $0.3 \mathrm{~Hz}$. On occasions, a typical tremor at rest persisted with similar amplitude and wave form when the arm was lifted into posture, then the frequency remained unchanged.

Several other Parkinsonian tremors could be grouped in the $6 \mathrm{~Hz}$ frequency band. The fine postural tremor, in appearance quite different from the resting tremor which can extend into posture, had a typical frequency between 6 and $6.2 \mathrm{~Hz}$. Intention tremor was an exaggeration of the postural tremor. A low frequency type of cogwheel phenomena occurred with a typical frequency of $6.2 \mathrm{~Hz}$. A low frequency type of action tremor which we refer to as active cogwheeling, occurred at a frequency indistinguishable from that of the passive cogwheel phenomenon. Finally, in some patients a sustained stretch at clinical feel of clonus which was also at $6 \mathrm{~Hz}$.

In the highest frequency band a few patients had postural tremors in the higher frequency type of cogwheel phenomena, and action tremor. Some also exhibited postural tremor between 7.6 and $9.4 \mathrm{~Hz}$. All patients who had active and passive "cogwheeling" at $6 \mathrm{~Hz}$ also had active and passive "cogwheeling" at 8 to $9 \mathrm{~Hz}$, 
which to avoid confusion and to communicate the clinical feel of the phenomenon we refer to as "rippling."

Gordon Holmes in 1904 commented on the similarity between the resting tremor of Parkinson's disease and the resting, postural and intention tremors of cerebello-rubal lesions. ${ }^{21}$ On the basis that they shared common mesencephalic structures and on the criterion of frequency, Holmes suggested that they shared a common mechanism. In our experience using spectral analysis to analyse cerebello-rubral tremors, we have found it impossible to distinguish them from Parkinsonian resting tremor on the basis of frequency alone. This is true not only for the upper limb, but also for the head. 1920

The tremor provided by sustained stretch in Parkinson's disease gives a clue to the possible origin of the $6 \mathrm{~Hz}$ tremor complex. It is indistinguishable from clonus as felt in patients with cervical cord lesions, and we see no reason why it should be referred to in any other terms. Yet it is found in patients with Parkinson's disease without clinical evidence of "pyramidal" lesion. The possible similarities between the tremor produced by sustained stretch in Parkinson's disease and clonus in "pyramidal" lesions was first commented upon by de Jong, ${ }^{9}$ and Denny-Brown ${ }^{3}$ asserted that "sudden stretch of the finger flexors will then, as also in the earlier stage of the disease, set up a series of the characteristic beats of tremor," a description which could be used to refer to the evocation of clonus. ${ }^{22}$ The mechanism responsible for the generation of the rhythm of clonus lies most probably in the spinal cord $;^{22}$ there is no apparent reason why the tremor of sustained stretch in Parkinson's disease should not be due to the same process. On the basis of similarities of wave form and frequency, we believe that the other $6 \mathrm{~Hz}$ tremors of the disease also involve the same mechanism.

Tremor in the region of $8-9 \mathrm{~Hz}$ occurs in a number of diseases. We have observed symptomatic postural tremor in this frequency band following cortical infarcts, with the tremor becoming asymptomatic with clinical recovery. In one case of mesencephalic angioma we observed a clonus tremor produced by sustained finger extension at a frequency of $9 \mathrm{~Hz}$ which was similar to the high frequency active rippling in Parkinson's disease. This frequency band is typical of normal physiological tremor, which is determined primarily by the minimum recruitment rate of motor units. ${ }^{23}$ Tremors in this frequency range may be caused by an exaggeration of normal physiological mechanisms. In the case of the high frequency clonus and rippling there must, in addition, be an unusually high degree of synchronisation of motor units.

\section{The identity of tremors at $6 \mathrm{~Hz}$ in Parkinson's disease}

Postural tremor at its most typical, intention tremor, low frequency active and passive cogwheeling and clonic tremor on sustained stretch in patients with Parkinson's disease all have frequencies close to $6 \mathrm{~Hz}$. This is only one feature which might suggest that they share a common mechanism. In addition, when several of these tremors occur in the same patient (for example fig 5), they have similar wave forms, and one can blend into another without alteration in rhythmicity. There is less similarity in wave forms between patients; however, typical wave forms which can be taken by cogwheel phenomena (figs 3,5 ), one of which is sinusoidal whilst the other consists of a fast stroke in one direction followed by a slower return, are also characteristic of clonus. With respect to power and harmonic distortion, the $6 \mathrm{~Hz}$ tremors lie on a continuum. Clonus is the most powerful of the tremors and has the most fixed frequency, whereas postural tremor is usually the weakest and has greatest harmonic distortion. Between these extremes, in descending order of power, lie action tremor, cogwheel phenomena and intention tremor. Thus we have two main differences separating these tremors, one being the way in which they are evoked and the other the degree of synchronisation and extent of resruitment and grouping of motor units. These several apparently diverse tremulous phenomena may have a common mechanism determining their rhythmicity. The tremogenic activity in each case is triggered in different ways; in clonus and cogwheel phenomena by peripheral input consequent upon stretch; and in action, intention and postural tremor by descending nervous activity either directly or as a consequence of stretch. Nevertheless once established the tremors are similar, differing largely only in magnitude and harmonic distortion.

\section{Clinical utility of tremor analysis in Parkinson's disease}

The common frequencies of symptomatic tremor in Parkinson's disease are equally $4-5 \mathrm{~Hz}$ and $6 \mathrm{~Hz}$. When occurring symptomatically they are more or less mutually exclusive. However, when asymptomatic tremor is taken into account, $75 \%$ of our patients had either a symptomatic resting tremor 
which on spectral analysis also had a smaller amplitude $6 \mathrm{~Hz}$ tremor either concurrent with the resting tremor or occurring in posture; or a symptomatic typical postural tremor at $6 \mathrm{~Hz}$ with a smaller asymptomatic $4-5 \mathrm{~Hz}$ tremor component. Such a combination of tremors at these two distinct frequencies in the same limb we have found only in basal ganglia disease, and accordingly consider this feature of the tremors of the disease to be pathognomonic. In support of this assertion, we have examined, using the same techniques, more than 25 patients with multiple sclerosis in which tremor was a symptom, six patients with peripheral neuropathy associated with tremor, four patients with tremor of "hysterical" origin, and eight patients with cerebrovascular disease or cerebral neoplasm producing tremor. Tremors were found at a variety of frequencies, but in these cases we have not found the combination of $4-5$ and $6 \mathrm{~Hz}$ tremors.

In elderly people presenting with tremor, the firm diagnosis of Parkinson's disease can be difficult and here the correct diagnosis is particularly important because of the possible serious side effects of anti-Parkinsonian drugs. If on spectral analysis the combination of $4-5$ and $6 \mathrm{~Hz}$ tremor is found (as occurred in $85 \%$ of our patients) then strong support is given to the diagnosis of Parkinson's disease. Conversely, when the diagnosis of basal ganglia disease is difficult, the inability to demonstrate two unrelated tremor frequencies in the same limb suggests alternative diagnoses. A clinical example of this situation is in the differential diagnosis of essential tremor occurring in elderly patients demonstrating a positive Froment sign; ${ }^{24}$ that is cogwheel phenomena detected in a limb only when the opposite limb is moved voluntarily. Although this sign was originally described as pathognomonic of Parkinson's disease, it is a frequent feature of uncomplicated essential tremor, thus making the distinction sometimes difficult. ${ }^{25}$

\section{Relationship between normal and pathological tremor}

Certain features of tremor in normal people are relevant to the analysis of tremors of Parkinson's disease. Our finding, in agreement with Marsden et $a l^{26}$ is that tremors in the right and left hands are frequently quite dissimilar. Tremor in the asymptomatic hand of a hemi-Parkinsonian patient cannot be used as representative of what the premorbid tremor would have been in the symptomatic limb.

It has been suggested by Lance et $a l^{10}$ that the action tremor of Parkinson's disease represents an exaggeration of normal physiological tremor. As the postural tremor, intention tremor and cogwheel phenomena of the disease are also similar in frequency, the question may be put as to whether they are all consequences of heightened normal physiological mechanisms. As shown, normal physiological tremor is a complex phenomenon, often composed of several frequencies of comparable significance. There is a tendency in normal tremor for a modal frequency of about $6 \mathrm{~Hz}$ with other modal frequencies between 7 and $9 \mathrm{~Hz}$. The $6 \mathrm{~Hz}$ tremors of Parkinson's disease may well represent an exaggeration of the particular component of normal tremor which contributes the content at $6 \mathrm{~Hz}$, but it would be simplistic and misleading to assert that they are an exaggeration of physiological tremor as if it were a unitary mechanism.

Further evidence that normal tremor has various component mechanisms is that under various conditions one or another aspect may well be heightened and made symptomatic. Thus, for example, tremor in the unstressed limb of a normal subject may be broadly tuned with significant components between 4 and $10 \mathrm{~Hz}$, whereas should the limb be stressed a more sharply tuned tremor at $6 \mathrm{~Hz}$ can appear (fig 9). Under prolonged stress of co-contraction a violent tremor develops: this, for example, is the common phenomenon of tremor in the limb of the fatigued and stressed rock climber; if occurring in pyramidal lesions it would be referred to as clonus and in Parkinson's disease "the tremor of sustained stretch." We believe that it is the same tremor in each case, the differences being only in the manner and circumstances of its evocation.

We thank the physicians at the National Hospital, Queen Square and at St Mary's Hospital, Paddington, for allowing us to study the patients under their care.

\section{References}

1 Parkinson J. An essay on the Shaking Palsy. London: Whitingham Rowland 1817.

2 Selby G. Parkinson's disease. In Vinken PJ, Bruyn GW, eds. Handbook of Clinical Neurology Vol 6, Diseases of Basal Ganglia. Amsterdam: North Holland 1968: 173-211.

3 Denny-Brown D. Clinical symptomatology of diseases of the basal ganglia. In: Vinken PJ, Bruyn GW, eds. Handbook of Clinical Neurology Vol 6, Diseases of Basal Ganglia. Amsterdam: North Holland 1968: 133-172. 
4 Struppler A, Erbel F, Velho F. Overview on the pathophysiology of Parkinsonian and other pathological tremors. In: Desmedt JE, ed. Physiological Tremor, Pathological Tremor and Clonus, Vol. 5, Progress in Clinical Neurophysiology. Basel: Karger 1978: 114-128.

5 Rondot P, Bathien N. Pathophysiology of Parkinsonian tremor. In: Desmedt JE, ed. Physiological Tremor, Pathological Tremor and Clonus Vol. 5, Progress in Clinical Neurophysiology. Basel: Karger 1978: 138-149.

6 Aronson N. Neurophysiology of tremor. J Neurosurg 1966; 24:207-9.

7 Ehringer H, Hornykiewicz O. Verteilung von Noradrenalin und Dopamine (3-hydroxytyramin) im Gehirn des Menschen und ihr Verhalten bei Erkrankungen des extrapyramidalen. Klin Weitschr 1960; 38:1236-9.

8 Lewy EF. Die Lehre vom Tonus und der Bewegung. Heidelberg: Springer 1923.

10 Lance JW, Schwarb RS, Peterson EA. Action tremor and the cogwheel phenomenon in Parkinson's disease. Brain 1963; 86:95-110.

11 Negro C. Le Phenomena “de la Roue Dentee". Encephale 1928; 23:203-4.

12 Moyer H. A new diagnostic sign in paralysis agitans, the cogwheel resistance of the extremities. JAMA 1911; 57:2125.

13 Matthews PBC, Rushworth G. Effect of Procaine on Stretch Reflex. J Physiol 1957; 135:245-64.

14 Matthews PBC, Rushworth G. The relative Sensitivity of Muscle Nerve Fibres to Procaine. J Physiol 1957; 135:65-9.

15 Denny-Brown D. Clinical symptomatology of diseases of the basal ganglia. In: Vinken PJ, Bruyn GW, eds. Handbook of Clinical Neurology Vol 6, Diseases of Basal Ganglia. Amsterdam: North Holland 1968: 133-72.

16 Stern J, Ward AA, Jr. The relationship of alpha and gamma motor systems to the efficiency of the surgical therapy of Parkinsonism. $J$ Neurosurg 1963; 20:185-7.

17 Jasper HH. Discussion on the anatomical and physiological aspects of Parkinson's disease. $J$ Neurosurg 1966; 24:235-6.

18 Evarts EV. Neurophysiological mechanisms in Parkinson's disease. In: Birkmayer W, Hornykiewicz $\mathrm{O}$, eds. Advances in Parkinsonism. Basel: Roche 1976: 37-51.

19 Findley LJ, Gresty MA, Halmagyi GM. A novel technique for recording arm movements and a survey of common abnormalities in nervous diseases. Arch Neurol 1980; 38:42.

20 Boshes B. Further insights into Parkinsonian tremor. In: Birkmayer W, Hornykiewcz $\mathrm{O}$, eds. Advances in Parkinsonism. Basel: Roche 1976: 303-18.

21 Holmes G. On certain tremors in organic cerebral lesions. Brain 1904; 27:360-75.

22 Dimitrijevic MR, Nathan PW, Sherwood AM. Clonus: The role of central mechanisms. J Neurol, Neurosurg and Psychiatry 1980; 43:321-32.

23 Marsden CD. The mechanisms of physiological tremor and their significance for pathological tremors. In: Desmedt JA, ed. Physiological Tremor, Pathological Tremor and Clonus Vol. 5, Progress in Neurophysiology. Basel: Karger 1978: 1-16.

24 Froment J, Gardere H. La rigidité et la rue dentée parkinsonienne s'efacent au répos. Rev Neurol 1926; 1:52-3.

25 Salisachs P. Dos Signos Clinicos no Conocidos del Tremblor Essencial. Med Clin (Barcelona) 1978; 70:120-1.

26 Marsden CD, Meadows JC, Lange GGW, Watson RS. The relation between physiological hand tremor of the two hands in healthy subjects. Electroencephalogr Clin Neurophysiol 1969; 27: 179-85. 